\title{
Nervous system and COVID-19
}

\author{
Bertha Torres-Oliva ${ }^{1}$, Karina Vélez-Jiménez², Idelfonso Rodríguez-Leyva ${ }^{3}$, and Lorena Guerrero-Torres ${ }^{4 *}$ \\ ${ }^{1}$ University of Guanajuato, Guanajuato; ${ }^{2}$ Hospital Ángeles Lomas, Mexico City; ${ }^{3}$ Neurology Service, Universidad Autónoma de San Luis Potosí, San \\ Luis Potosi; ${ }^{4}$ Department of Infectious Diseases, Instituto Nacional de Ciencias Médicas y Nutrición Salvador Zubirán, Mexico City. Mexico
}

A new disease has been reported recently: COVID-19, caused by a novel virus which was named SARS-CoV-2 coronavirus'. COVID-19 started in Wuhan, China, in late December 2019 at a seafood market. Due to its high rate contagion, 59 suspected patients were transferred to a designated hospital in Wuhan, China. The predominant symptoms were fever, dry cough, myalgia, fatigue, and, less frequently, headache, hemoptysis, and diarrhea. More than half of the patients developed dyspnea during the $2^{\text {nd }}$ week of the onset of symptoms and all presented pneumonia with abnormal chest CT, as well as lymphopenia. A significant elevation in inflammatory markers was found in patients admitted to the intensive care unit (ICU). In 41 of the 59 cases, nCoV 2019 infection was confirmed by next-generation sequencing or real-time RT-PCR methods'.

In this case series, a third of the patients had underlying diseases such as diabetes, hypertension, and cardiovascular disease. Furthermore, it was more common in males $(73 \%)$, with a median age of 49 years. Other studies have confirmed that age and comorbidities are associated with higher rates of hospitalization for COVID-192,3.

Regarding the neurological manifestations of COVID-19 infection, in the retrospective study published by Mao et al., the presence of neurological symptoms was sought intentionally in 214 hospitalized patients with confirmed SARSCoV-2 disease. They were classified into three categories: (1) signs and symptoms of affection to the central nervous system (CNS) such as headache, dizziness, alteration of consciousness, ataxia, acute cerebrovascular disease, and epilepsy, (2) signs and symptoms of affection to the cranial and peripheral nerves, such as taste impairment, smell impairment, vision impairment, or neuralgia, and (3) skeletal and muscular injury manifestations. Neurological manifestations were found in 78 (36.4\%) of patients. CNS symptoms were the main form of neurological injury, present in $53(24.8 \%)$ of the cases. The most common symptoms were dizziness (16\%) and headache (13\%). Cranial nerve involvement was infrequent, with hypogeusia in $12(5.6 \%)$, hyposmia in $11(5.1 \%)$, and vision problems in $3(2.3 \%)$. There were $23(10.7 \%)$ patients with muscle injury ${ }^{2}$.

This study also showed differences between the characteristics of patients with severe infection $(41 \%)$ and nonsevere infection (58.9\%). Patients with severe infection were significantly older (58.2 \pm 15.0 vs. 48.9 $\pm 14.7 ; p<0.001)$, had more comorbidities $(47.7 \%$ vs. $32.5 \% ; p<0.05)$, especially hypertension $(36.4 \%$ vs. $15.1 \% ; p<0.001$ ), and had fewer typical symptoms compared to patients with nonsevere infection. Furthermore, neurological disease was more common in patients with severe infection compared with nonsevere infection ( $45.5 \%$ vs. $30.2 \% ; p<0.02)$, particularly acute cerebrovascular disease $(5.7 \%$ vs. $0.8 \% ; p<0.001)$, altered consciousness ( $14.8 \%$ vs. $2.4 \% ; p<0.001)$, and skeletal muscle damage (19.3\% vs. $4.8 \% ; p<0.001)^{2}$.

Although neurological manifestations of COVID-19 seemed uncommon, the self-reported olfactory and taste disorders (OTD) questionnaire carried out by Giacomelli

\section{Correspondence:}

*Lorena Guerrero-Torres

Department of Infectious Diseases

Instituto Nacional de Ciencias Médicas y Nutrición Salvador Zubirán

Vasco de Quiroga 15, Tlalpan

Date of reception: 19-04-2020

Date of acceptance: 22-04-2020

DOI: 10.24875/RMN.M20000079
Available online: $18-05-2020$ Mex Neuroci. 2020;21(3):80-81 www.revmexneurociencia.com

E-mail: Iorena.guerrero.torres@gmail.com

Permanyer. This is an open access article under the CC BY-NC-ND license 1665-5044/@ 2020 Academia Mexicana de Neurolog
(http://creativecommons.org/licenses/by-nc-nd/4.0/). 
et al. in 59 hospitalized patients with COVID-19 in Milan, Italy, revealed the necessity to expand the study of these manifestations in nonhospitalized infected patients with COVID-19. These investigators reported an alteration in taste or smell in 20 (33.9\%) of patients and the presence of both symptoms in $11(18.6 \%)$ of the patients. Furthermore, $20.3 \%$ of the patients experienced the symptoms before hospital admission and $13.5 \%$ after hospital admission. Changes in taste were frequently (91\%) identified before hospitalization. Moreover, OTDs were more common in women and younger people ${ }^{4}$.

In a recent report of 58 patients hospitalized for SARS-CoV-2 acute respiratory distress syndrome, neurological abnormalities were detected in 49 (84\%) of them; 40 (69\%) presented agitation and confusion, and $39(67 \%)$ a corticospinal tract injury. Magnetic resonance image of the brain performed in 13 patients revealed meningeal reinforcement in $8(62 \%)$, cerebrovascular disease in $3(23 \%)$, and perfusion abnormalities in 11 (100\%) patients who underwent this sequence. In the cerebrospinal fluid (CSF) examination of seven patients who underwent lumbar puncture, the presence of oligoclonal bands was found in $2(29 \%)$, high proteins and IgG in 1 (14\%), and low albumin in 4 (57\%). All CSF samples were negative for SARS-CoV-2 ${ }^{5}$.

Other manifestations caused by SARS-CoV-2 have emerged. As reported by Italian researchers, five patients with COVID-19 among their 1000 to 12,000 patients presented a typical Guillain-Barré syndrome (GBS), with an interval from 5 to 10 days between the onset of COVID-19 symptoms and the first Guillain-Barré symptom. Three of these patients had ageusia or anosmia 5-7 days before the start of GBS. The CSF analysis reported an average protein level in two of them and a normal leukocyte count in all patients. Antiganglioside antibodies were tested in three patients and found normal. All RT-PCR of the CSF was negative for SARS-CoV-2. The electrophysiological studies reported an axonal variant in three patients and a demyelination variant in two patients. Gadolinium-based MRI showed an enhancement of the caudal nerve roots in two patients, enhancement of the facial nerve in one patient, and no signal change in nerves in two of them ${ }^{6}$.

Angiotensin-converting enzyme 2 (ACE-2) receptors have recently been identified as the site of entry into the cell by the SARS-CoV-2 virus ${ }^{7}$. These receptors are present in multiple organs such as the lung, the nervous system, and skeletal muscle, such that SARS-CoV-2 can cause neurological symptoms by direct or indirect mechanisms $s^{7-9}$. These receptors have been detected in glial cells and neurons, making it a potential target for SARS-CoV- ${ }^{8}$. The pathogenic mechanism for invading the central nervous system of SARS-CoV-2 is suspected to be hematogenous or the retrograde neuronal pathway ${ }^{2,7,8}$.

The proximity of the cribriform plate to the olfactory bulb could enable the SARS-CoV-2 virus to reach and inflict cerebral damage ${ }^{8}$. Therefore, changes in smell (hyposmia) could appear as an early symptom in the uncomplicated stage of COVID-194,8. Similarly, ACE-2 receptors are used by the SARS-Cov2 virus to penetrate the epithelial cells of the mucosa of the oral cavity and on the tongue. These findings could explain the pathogenic mechanism in the alterations of taste and odor in SARS-CoV-2 infection ${ }^{10}$.

On March 11, 2020, the World Health Organization (WHO) declared the COVID-19 as a pandemic due to the exponential increase in cases outside of China, the number of countries affected, and the high mortality. To date, 2,319,066 confirmed cases had been reported in 213 countries, with 157,970 deaths worldwide ${ }^{11}$.

Humanity faces the most significant challenge in 100 years: the appearance of this new COVID-19 caused by a novel virus capable of affecting all the organs and systems, especially the lungs. Increasing evidence shows that SARS-CoV-2 may also invade the CNS and cause neurological manifestations. Damage to the nervous system can come in different forms and severity. Sudden loss of smell and taste as a symptom of infection should be further studied, as it could be a screening tool that contributes to early diagnosis and timely isolation. Innovating and learning about this disease is one of the critical areas to prevent infections, save lives, and hinder its effects.

\section{References}

1. Huang C, Wang Y, Li X, Ren L, Zhao J, Hu Y, et al. Clinical features of patients infected with 2019 novel coronavirus in Wuhan, China. Lancet. 2020;395:497-506.

2. Mao L, Wang M, Chen S, Jin $\mathrm{H}, \mathrm{Hu} Y, \mathrm{He} Q$, et al. Neurologic manifestations of hospitalized patients with coronavirus disease 2019 in Wuhan, China. JAMA Neurol. 2020 [Epub ahead of print].

3. Guan W, Ni Z, Hu Y, Liang W, Ou C, He J, et al. Clinical characteristics of coronavirus disease 2019 in China. N Engl J Med. 2020;382:1708-20.

4. Giacomelli A, Pezzati L, Conti F, Bernacchia D, Siano M, Oreni L, et al. Self-reported olfactory and taste disorders in SARS-CoV-2 patients: a cross-sectional study. Clin Infect Dis. 2020 [Epub ahead of print].

5. Helms J, Kremer S, Merdji H, Clere-Jehl R, Schenck M, Kummerlen C, et al. Neurologic features in severe SARS-CoV-2 infection. N Engl J Med. 2020 [Epub ahead of print].

6. Toscano G, Palmerini F, Ravaglia S, Ruiz L, Invernizzi P, Cuzzoni MG, et al. Guillain-Barré syndrome associated with SARS-CoV-2. N Engl J Med. 2020 [Epub ahead of print].

7. Wu Y, Xu X, Chen Z, Duan J, Hashimoto K, Yang L, et al. Nervous system involvement after infection with COVID-19 and other coronaviruses. Brain Behav Immun. 2020 [Epub ahead of print].

8. Baig AM, Khaleeq A, Ali U, Syeda H. Evidence of the COVID-19 virus targeting the CNS: tissue distribution, host-virus interaction, and proposed neurotropic mechanisms. ACS Chem Neurosci. 2020;11:995-8.

9. LiYC, Bai WZ, Hashikawa T. The neuroinvasive potential of SARS-CoV2 may play a role in the respiratory failure of COVID-19 patients. J Med Virol. 2020:92:552-5.

10. Xu H, Zhong L, Deng J, Peng J, Dan H, Zeng X, et al. High expression of ACE2 receptor of 2019-nCoV on the epithelial cells of oral mucosa. Int J Oral Sci. 2020;12:8.

11. Available from: https://www.who.int/emergencies/diseases/novel-coronavirus-2019 [Accessed Apr 22, 2020].. 